\title{
Charge balancing and the fall off of the ridge
}

\author{
Piotr Bożek ${ }^{\mathrm{a}, \mathrm{b}}$, Wojciech Broniowski ${ }^{\mathrm{b}, \mathrm{c}}$ \\ ${ }^{a}$ AGH University of Science and Technology, Faculty of Physics and Applied Computer Science, al. Mickiewicza 30 \\ 30-059 Krakow, Poland \\ ${ }^{b}$ Institute of Nuclear Physics PAN, ul. Radzikowskiego 152, 31-342 Krakow, Poland \\ ${ }^{c}$ Institute of Physics, Jan Kochanowski University, 25-406 Kielce, Poland
}

\begin{abstract}
Two-dimensional correlation functions in $\Delta \eta-\Delta \phi$ for charged hadrons emitted in heavy-ion collisions are calculated in event-by-event hydrodynamics. With the Glauber model for the initial density distributions in the transverse plane and elongated density profiles in the longitudinal direction, the flow patterns in the azimuthal angle of the two-dimensional correlation function are properly reproduced. We show that the additional fall-off of the same-side ridge in the longitudinal direction can be explained as an effect of local charge conservation at a late stage of the evolution. This additional non-flow effect increases the harmonic flow coefficients for the unlike-sign particle pairs.
\end{abstract}

\section{Introduction}

The collective expansion of dense matter in heavy-ion collisions is determined by the geometry of the fireball. In the longitudinal direction the density profile is assumed to be approximately boost-invariant for central rapidities, while in the transverse plane it is asymmetric. The shape and the size of the fireball fluctuates event-by-event [1-3]. The dependence of the correlation function on the azimuthal angle is dominated by the even and odd harmonic flow components [1, 4, 5] and can be reproduced in event-by-event viscous hydrodynamics calculations [6-8]. The shape of the same-side ridge in the longitudinal (pseudorapidity $\eta$ ) direction can yield important information on the mechanism of the energy deposition in the early stage of the collision [9-13]. The dominant features observed in the data are the same-side $(\Delta \phi \simeq 0)$ and away-side $(\Delta \phi \simeq \pi)$ ridge, with the latter one exhibiting no dependence on $\Delta \eta$, and the former one showing an increase of the correlation function in the form of a same-side peak. This structure is much more pronounced for correlations of the unlike-sign hadrons. [14, 15].

The formation of charges at a late stage of the collective evolution induces strong correlations between the unlike-sign hadrons in the pseudorapidity and azimuthal angle [16-19]. We argue that the same mechanism explains the observed shape of the same-side ridge in the (unbiased) two-dimensional correlation function [20]. These non-flow correlations from the local charge conservation also yield a small contribution to the flow coefficients.

\section{Hydrodynamic model with local charge conservation}

We use a 3+1-dimensional viscous hydrodynamic model [21] with bulk and shear viscosities [22]. The calculations are run event-by-event with the initial entropy density generated as coarse- 
grained distributions from GLISSANDO [23]. Particle emission at freeze-out is performed using the THERMINATOR code [24].

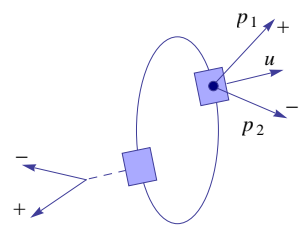

Figure 1: Mechanism of the generation of charge conservation correlations from resonance decays and local pair creation.

We implement the local charge conservation in the statistical emission code. Opposite-charge particles are emitted in pairs from the same fluid element. Thus, they feel the same collective flow velocity which collimates their motion. The spread in their relative momenta comes the thermal motion (Fig. 11). The procedure used to generate particle distributions presented here yields slightly stronger correlations compared to [20], including pairs from resonance decay cascades as well. Our hydrodynamic model reproduces well the particle spectra and flow coefficients.

\section{Two-dimensional correlation functions}

(a) $30-40 \%$, unbal.

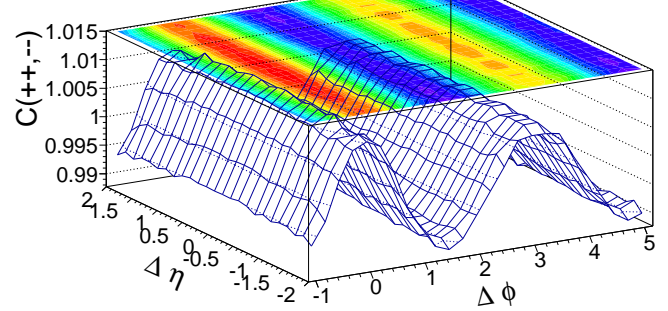

(c) $30-40 \%$, bal.

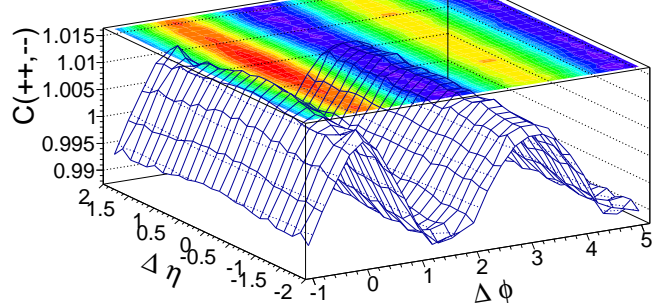

(b) $\quad 30-40 \%$, unbal.

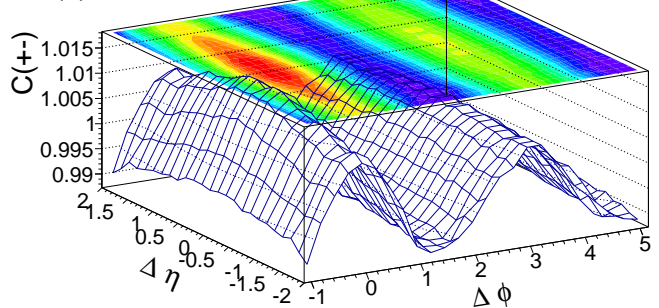

(d) $30-40 \%$, bal.

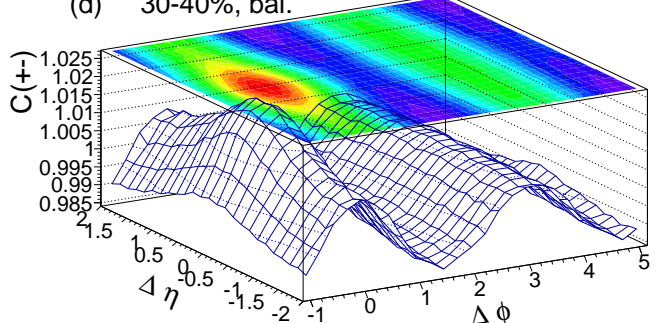

Figure 2: Two-dimensional correlation functions for particle emission without local charge conservation (panels (a) and (b)) and with local charge conservation (panels (c) and (d)), Au-Au collisions at $\sqrt{s_{N N}}=200 \mathrm{GeV}$ in $30-40 \%$ centrality class $\left(T_{f}=150 \mathrm{MeV}, 0.2<p_{T}<2 \mathrm{GeV}\right)$. 
Two-dimensional correlation functions

$$
C(\Delta \eta, \Delta \phi)=N_{\text {real }}^{\mathrm{pair}}(\Delta \eta, \Delta \phi) / N_{\text {mixed }}^{\mathrm{pair}}(\Delta \eta, \Delta \phi),
$$

are calculated for the like- and unlike-sign hadron pairs. In the case of the uncorrelated statistical emission of particles at freeze-out there is only a small difference in the results for different charge combinations (panels (a) and (b) in Fig. 2). Some weak short range correlations between unlike charged particles come from resonance decays. The same-side peak in the unlike-sign correlation function (panel (b)) is much smaller than observed experimentally.

The local charge conservation mechanism generates noticeable correlations in the directions of the emitted hadron pairs. The unlike-sign particle pairs generated at freeze-out are collimated by the common collective flow. The effect is clearly seen as a strong same-side peak in the unlike-sign correlation function (panel d)) that is not observed for like-sign pairs (panel c)). These features of the same-side ridge are compatible with the experiment [14].

\section{Flow coefficients}

The two-dimensional correlation function shown in Fig. 2 contains the information on all the harmonic flow coefficients. The local charge conservation effect gives a non-flow contribution to the observed $v_{n}^{2}$ coefficients. The charge conservation correlations decrease with the multiplicity and with the pseudorapidity separation of the particle pair (Fig. 3). The non-flow correlation increase $v_{n}^{2}$ at small pseudorapidity separations, in a similar way as the resonance decays. The mechanism of local charge conservation explains the magnitude and the range in $\Delta \eta$ of the nonflow correlations in $v_{n}$ and is consistent with the measured difference of the flow coefficients for unlike and like-sign pairs.
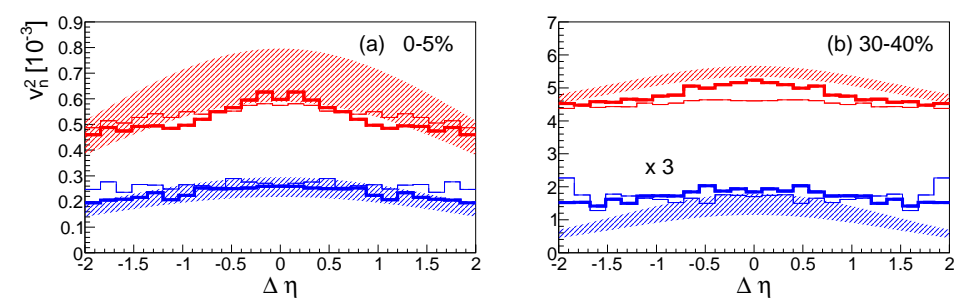

Figure 3: Elliptic (upper lines) and triangular (lower lines) flow coefficients plotted as function of the relative pseudorapidity of the pair. The thick and thin lines represent the results of the simulations with and without the local charge conservation, respectively $\left(T_{f}=150 \mathrm{MeV}, 0.15<p_{T}<4 \mathrm{GeV}\right)$. The shaded bands are extracted from the measured two-dimensional correlation functions [14].

Figure 4 shows the $p_{\perp}$ dependence of $v_{2}$. The local charge conservation effects give a small contribution to the $v_{2}$ measured with the second cumulant method. These effects may be reduced when using a pseudorapidity gap for the pair or a $Q$-vector defined at forward rapidities. The mechanism of local charge conservation presents a dominant source of non-flow correlation in heavy-ion collisions. It gives a noticeable contribution to $v_{1}$ and $v_{2}$. The charge splitting induced by the local charge conservation effects in $v_{1}$ gives a large contribution to charge parity violation signals [25, 26]. However, to reproduce the magnitude of the charge-independent correlations [27], the total transverse momentum conservation has to be imposed [28]. Within the hydrodynamic model with flow and local charge conservation the charge balance functions in relative 
pseudorapidity can be extracted as well. The results are in satisfactory agreement with the data [19].
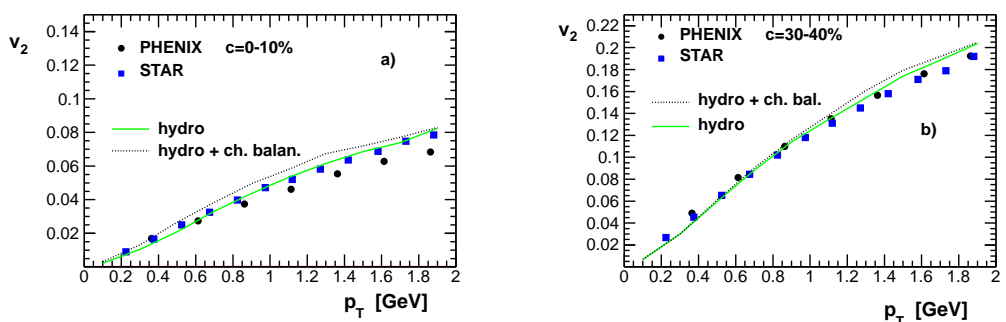

Figure 4: The elliptic flow coefficient of charged particles with (dashed lines) or without (solid lines) local charge conservation mechanism, centrality 0-10\% (panel a) and 30-40\% (panel b). Data from [29, 30].

Supported by Polish Ministry of Science and Higher Education, grant N N202 263438, and National Science Centre, grant DEC-2011/01/D/ST2/00772.

\section{References}

\section{References}

[1] J. Takahashi, B. Tavares, W. Qian, R. Andrade, F. Grassi, et al., Phys. Rev. Lett. 103 (2009) 242301.

[2] B. Alver, G. Roland, Phys. Rev. C81 (2010) 054905.

[3] W. Broniowski, M. Chojnacki, L. Obara, Phys. Rev. C80 (2009) 051902.

[4] M. Luzum, Phys.Lett. B696 (2011) 499.

[5] K. Werner, I. Karpenko, M. Bleicher, T. Pierog, S. Porteboeuf-Houssais, Phys.Rev. C85 (2012) 064907.

[6] B. Schenke, S. Jeon, C. Gale, J.Phys.G G38 (2011) 124169.

[7] Z. Qiu, C. Shen, U. Heinz, Phys. Lett. B707 (2012) 151.

[8] P. Bozek, W. Broniowski, Phys. Rev. C85 (2012) 044910.

[9] S. Gavin, L. McLerran, G. Moschelli, Phys. Rev. C79 (2009) 051902.

[10] F. Gelis, T. Lappi, R. Venugopalan, Nucl. Phys. A830 (2009) 591c.

[11] K. Dusling, T. Epelbaum, F. Gelis, R. Venugopalan, arXiv:1203.5704 [nucl-th] (2012).

[12] S. Gavin, G. Moschelli, Phys. Rev. C85 (2012) 014905.

[13] J. Kapusta, B. Muller, M. Stephanov, Phys. Rev. C85 (2012) 054906.

[14] G. Agakishiev, et al., arXiv:1109.4380 [nucl-ex] (2011).

[15] B. Abelev, et al., arXiv:0806.0513 [nucl-ex] (2008).

[16] S. A. Bass, P. Danielewicz, S. Pratt, Phys. Rev. Lett. 85 (2000) 2689-2692.

[17] S. Jeon, S. Pratt, Phys. Rev. C65 (2002) 044902.

[18] P. Bozek, Phys.Lett. B609 (2005) 247.

[19] M. M. Aggarwal, et al., Phys. Rev. C82 (2010) 024905.

[20] P. Bozek, W. Broniowski, Phys.Rev.Lett. 109 (2012) 062301.

[21] P. Bożek, Phys. Rev. C85 (2012) 034901.

[22] P. Bożek, Phys. Rev. C81 (2010) 034909.

[23] W. Broniowski, M. Rybczyński, P. Bożek, Comput. Phys. Commun. 180 (2009) 69.

[24] M. Chojnacki, A. Kisiel, W. Florkowski, W. Broniowski, Comput. Phys. Commun. 183 (2012) 746.

[25] S. Schlichting, S. Pratt, Phys.Rev. C83 (2011) 014913.

[26] Y. Hori, T. Gunji, H. Hamagaki, S. Schlichting, arXiv:1208.0603 [nucl-th] (2012).

[27] B. Abelev, et al., Phys.Rev. C81 (2010) 054908.

[28] A. Bzdak, V. Koch, J. Liao, arXiv:1207.7327 [nucl-th] (2012).

[29] J. Adams, et al., Phys. Rev. C72 (2005) 014904.

[30] A. Adare, et al., Phys.Rev.Lett. 107 (2011) 252301. 